\title{
Space charge polarization in irradiated single crystal CVD diamond
}

Naaranoja, T.

2019-06

Naaranoja, T , Golovleva, M , Martikainen , L , Berretti , M \& Österberg , K 2019 , ' Space

charge polarization in irradiated single crystal CVD diamond ', Diamond and Related

Materials , vol. 96 , pp. 167-175 . https://doi.org/10.1016/j.diamond.2019.03.007

http://hdl.handle.net/10138/327967

https://doi.org/10.1016/j.diamond.2019.03.007

cc_by_nc_nd

acceptedVersion

Downloaded from Helda, University of Helsinki institutional repository.

This is an electronic reprint of the original article.

This reprint may differ from the original in pagination and typographic detail.

Please cite the original version. 


\section{Accepted Manuscript}

Space charge polarization in irradiated single crystal CVD diamond

T. Naaranoja, M. Golovleva, L. Martikainen, M. Berretti, K. Österberg

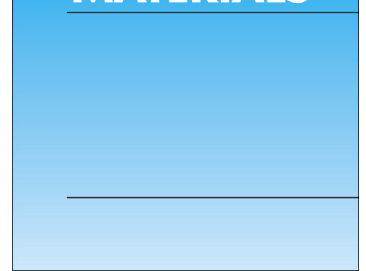

PII:

S0925-9635(18)30766-0

DOI: https://doi.org/10.1016/j.diamond.2019.03.007

Reference:

DIAMAT 7363

To appear in:

Diamond \& Related Materials

Received date:

31 October 2018

Revised date:

5 February 2019

Accepted date:

11 March 2019

Please cite this article as: T. Naaranoja, M. Golovleva, L. Martikainen, et al., Space charge polarization in irradiated single crystal CVD diamond, Diamond \& Related Materials, https://doi.org/10.1016/j.diamond.2019.03.007

This is a PDF file of an unedited manuscript that has been accepted for publication. As a service to our customers we are providing this early version of the manuscript. The manuscript will undergo copyediting, typesetting, and review of the resulting proof before it is published in its final form. Please note that during the production process errors may be discovered which could affect the content, and all legal disclaimers that apply to the journal pertain. 


\title{
Space charge polarization in irradiated single crystal CVD diamond
}

\author{
T. Naaranoja ${ }^{1}$, M. Golovleva, L. Martikainen, M. Berretti, K. Österberg \\ Helsinki Institute of Physics, Gustaf Hällströmin katu 2, P.O. Box 64, FI-00014 University of \\ Helsinki, Finland \\ Department of Physics, Gustaf Hällströmin katu 2, P.O. Box 64, FI-00014 University of \\ Helsinki, Finland \\ Lappeenranta University of Technology, Skinnarilankatu 34, 53850 Lappeenranta, Finland
}

\begin{abstract}
Single crystal CVD (scCVD) diamond is an attractive material for particle detection in high energy physics for its good time resolution and reported outstanding radiation tolerance. In addition to direct signal loss via charge carrier trapping, polarization effect, caused by non-homogeneous filling of trap defects, is a known cause of signal degradation in irradiated scCVD diamond. This phenomenon was studied by intentionally polarizing irradiated diamonds. Even the relatively lightly irradiated $\left(10^{14}\right.$ protons $\left./ \mathrm{cm}^{2}\right)$ diamonds exhibited strong enough polarization to collapse the electric field with moderate rate of $5 \mathrm{MeV}$ alpha particles. The transient current measurements were reproduced with TCAD simulations. The hypothesis that the polarization is caused by single neutral defect type in the bulk, was tested using two generic models. Neither one has a satisfactory agreement with the measurement data, which indicates that trapping at the interfaces play a significant role in space charge polarization.
\end{abstract}

Keywords single crystal diamondradiation induced effects, electrical properties characterization, defects, detectors, sensors

\section{Introduction}

Diamonds are an attractive material for radiation detectors first of all due to their outstanding radiation tolerance [1] and excellent time resolution, when measuring the time-of-flight for hadrons [2]. Its wide band gap results in high resistivity and small leakage currents in diamond-based detectors. Hence, diamond has found its way to such harsh radiation environments as experiments at CERN's Large Hadron Collider and is currently used in CMS-TOTEM Precision Proton Spectrometer [3] as time-of-flight detector and previously in TOTEM timing upgrade [4].

Radiation damage in scCVD diamond is currently explained by introduction of neutral vacancy defects that affect both holes and electrons collection. Slightly higher trapping probability of electrons than holes has previously been observed [5,6,7]. Polarization under irradiation with heavy ions, has reportedly higher trapping probability for holes [8].

However, it has been observed that low fluence laboratory studies and high fluence in situ experience differ from each other [5]. One possible explanation for the difference is the polarization due to charge trapping, which is more easily avoided in laboratory than in a particle physics experiment, where the particle flux is not a controlled parameter. Polarization

\section{${ }^{1}$ Corresponding author}

Email address: tiina.naaranoja@ @elsinki.fi (T. Naaranoja) 
occurs as build-up of charge inside detector's bulk that eventually reduces charge collection efficiency (CCE) of the detector. It can be countered by either filling all traps, e.g. by irradiating with an beta-source or with UV light, or periodically releasing trapped charge e.g. by periodically switching bias voltage on and off.

Similar effects might arise from charge trapping in the diamond-metal interface. Such Schottky contact would also enhance the trapping of charge in bulk defects. To avoid polarization effectively in the experiments, the mechanism leading to it needsto be understood.

The detector design in this study corresponds to the largest pixel size that has been used in the TOTEM and PPS timing detectors. This corresponds to the worst case scenario for interface trapping in these experiments. Especially the efficacy for mitigating polarization by switching bias voltage on and off was investigated since it is easy method to adapt in experiments. The polarization in this study is expected to have contribution both from the non-homogeneous trapping in the diamond bulk, trapping at the interfaces and interface potential enhanced trapping in bulk. As the bulk defects are often assumed to be the sole contributor to polarization and bulk defects are relatively well known, polarization originating on trapping in the bulk only is used as the null hypothesis.

\section{Material and methods}

$\mathrm{R} 0.2$

\section{Samples}

$$
\begin{aligned}
& \text { ThicknessElectrode Fluence } \\
& (\mu \mathrm{m}) \quad \text { (protons } / \mathrm{cm}^{2} \text { ) } \\
& 340 \pm 10 \quad \text { TiW } \quad(1.10 \pm \\
& 340 \pm 10 \mathrm{Cr} / \mathrm{Au} \quad \begin{array}{c}
0.08) \times 10^{14} \\
(0.97 \pm
\end{array} \\
& \begin{array}{ll}
540 \pm 10 \quad \mathrm{TiW} & (1.06 \pm
\end{array} \\
& 540 \pm 10 \quad \operatorname{TiW} \quad \begin{array}{l}
0.07) \times 10^{14} \\
(4.5 \pm 0.3) \times
\end{array} \\
& 10^{15} \\
& 540 \pm 10 \mathrm{Cr} / \mathrm{Au} \quad(4.4 \pm 0.3) \times \\
& 10^{15}
\end{aligned}
$$

Table 1. Summary of the scCVD diamond samples

The diamond substrate for this study was procured from Element Six [9]. The used material was the highest purity single crystal Chemical Vapor Deposition (scCVD) diamond available, so-called "electronic grade". For the electrodes a simple test pattern was used: single $4.2 \times$ $4.2 \mathrm{~mm}^{2}$ square pad on a $4.5 \times 4.5 \mathrm{~mm}^{2}$ diamond surface. For the electrodes two materials were used: TiW produced by Princeton [10] and Cr/Au by Applied Diamond [11]. The samples are summarized in Table 1 and one of the samples is depicted in Figure 2.

\section{Irradiation}

Five single crystal diamonds were irradiated at CERN IRRAD facility with $24 \mathrm{GeV} / \mathrm{c}$ protons, three to a target fluence of $10{ }^{14}$ protons $/ \mathrm{cm}^{2}$ and two to $5 \times 10^{15}$ protons $/ \mathrm{cm}^{2}$. The dosimetry results for all irradiation in 2017 are publicly available [12]. The standard beam 
parameters were used and the full width at half maximum of the Gaussian beam was $14.0 \pm$ $0.4 \mathrm{~mm}$ in vertical direction and $14.3 \pm 0.07 \mathrm{~mm}$ in horizontal direction at the sample location. The fully processed diamond detectors were irradiated with bias in ambient conditions and the beam induced currents in diamond were monitored.

\section{Transient Current Technique}

After irradiation the diamond detectors were studied with a mixed nuclide $\alpha$-source with nuclides (and $\alpha$ particle energies) Am-241 (5.486 MeV), Cm-244 (5.805 MeV) and Pu-239 $(5.156 \mathrm{MeV})$ in equal quantities. The particle energies are below the level where plasma effect is expected i.e. single interaction does not inject enough charge to significantly perturb the

electric field. The $\alpha$ particles deposit charge close to the surface, which allows the investigation of the hole and electron transport properties separately. One type of charge carrier is almost immediately collected at the closest electrode and the other drifts through the bulk to the other electrode. Using Shockley-Ramo theorem $[13,14]$, the electric field inside the detector can be deduced.

The bias voltage was supplied using a Keithley 2410 SourceMeter unit. A single-channel

readout electronics system was used. It consisted of a Particulars AM-01 A broadband amplifier, that has a bandwidth of $10 \mathrm{kHz}-2 \mathrm{GHz}$ and gain of $53 \mathrm{~dB}$, and a Particulars bias $\mathrm{T}$ with bandwidth of $100 \mathrm{kHz}-2 \mathrm{GHz}$. The signal was read out using a LeCroy WavePro 7300A 3

$\mathrm{GHz}$ digital oscilloscope and a custom-written data acquisition software. For the used oscilloscope settings the sampling period was 100 ps and jitter 1 ps.

The $\alpha$ source was placed at a $2 \mathrm{~mm}$ distance from the diamond, which is as close as possible without touching in the transient current technique (TCT) setup in order to achieve as strong space charge polarization as possible. The estimated particle rate was $5000 \pm 800$ particles/s at the detector surface in an area of $A_{i r r}=(14 \pm 3) \mathrm{mm}^{2}$. The maximum observed hit rate was restricted to about 14 events/s by the readout system.

The radiation source was placed on the diamond and bias voltage was ramped up with speed of

$200 \mathrm{~V} / \mathrm{s}$. The time for polarization was recorded from turning on the bias voltage. After reaching the polarized state, the bias voltage was turned off until the polarization had fully dissipated and the measurement was repeated 5 to 10 times. The initial state before polarization was recorded during the first 10 seconds after turning bias on.

\section{TCAD simulations}

In order to investigate and understand the scCVD diamond bulk internal properties better, numerical simulations of the initial state before the polarization were carried out using Synopsys Sentaurus Technology Computer-Aided Design (TCAD) software eSynopsys:2018.

The simulated device structure had dimensions $(100 \times 300 \times 1) \mu \mathrm{m} \quad{ }^{3}$, where $300 \mu \mathrm{m}$ corresponds to the device thickness, and it was scaled with an area factor $A$ to match the dimensions of the real device. In the simulation the diamond bulk was considered with the concentration $n_{i}=10^{-27} \mathrm{~cm}{ }^{3}$ and Schottky type contacts with a low barrier height of 0.8 $\mathrm{eV}$. At present this material is not included in the material libraries of TCAD simulation tools, therefore, all the physical parameters of the scCVD diamond, such as permittivity, optical refractive index and band gap, were defined by hand using data from Refs. [16,17]. The band gap energy used was $5.47 \mathrm{eV}$ with electron affinity $1.73 \mathrm{eV}$ for the oxygen-terminated diamond surfaces.

Nitrogen and boron are known donor and acceptor species in diamond. In order to take into account the effect of these impurities, two impurity levels were introduced into the diamond 
band gap: acceptor and donor type levels with energies $E_{v}+0.368 \mathrm{eV}$ and $E_{c}-1.7 \mathrm{eV}$, respectively, each having concentration $10^{12} \mathrm{~cm}^{-3}$. The concentration was let vary between the maximum concentration $10^{14} \mathrm{~cm}^{-3}$ declared by the producerredblack [9]black and the minimum concentration required for stable simulation $10^{12}$ $\mathrm{cm}{ }^{-3}$. The used concentration $10^{12} \mathrm{~cm}^{-3}$ is the best fitting value in this regime. The impurities' electron $\sigma_{e}=10^{-14} \mathrm{~cm}^{2}$ and hole $\sigma_{h}=10^{-13} \mathrm{~cm}{ }^{2}$ capture cross sections were found by fitting simulated transients to the measurement results. The obtained cross section for electron in boron is in good agreement with the experimentally measured value [18], but nitrogen has larger obtained cross section than found experimentally [18]. The mobilities were determined experimentally in Section 1 and used as an input parameter in the simulations.

Transient analysis with $\alpha$-particles was performed on the described above structure and then compared with the corresponding measurements carried out on real devices stimulated with alpha particles. The $\alpha$-particle interaction was simulated using the inbuilt Heavy Ion Model with $12 \mu \mathrm{m}$ radiation length.

To reproduce the TCT data for irradiated samples, a neutral level with energy $E_{v}+2.85 \mathrm{eV}$ corresponding to a neutral mono-vacancyredblack [19] black was introduced. A good agreement with the experimental data was obtained by tuning the defect concentration to $10^{14}$ $\mathrm{cm}^{-3}$, which was also measured in section 1 , and electron and hole capture cross sections to $\sigma_{e}=1 \times 10^{-14} \mathrm{~cm}^{2}$ and $\sigma_{h}=5 \times 10^{-15} \mathrm{~cm}^{2}$, respectively. When compared to measured defect concentrations for $26 \mathrm{MeV}$ proton irradiated diamonds by Pomorski redblack [20]black, defect concentration in the order of $10^{1} 4 \mathrm{~cm}^{-3}$ is expected. Capture cross section close to the order of $10^{-15} \mathrm{~cm}^{2}$ is also expected for neutral defectsredblack [21]black.

\section{Theory}

\section{Current transient form}

The form of the current transient follows the electric field inside the detector according to the Shockley-Ramo theorem $[13,14]$. The induced current for single charge carrier is

$$
i(x)=q v \cdot E_{0}(x) \text {, }
$$

where $q$ is the elementary charge, $v$ is the instantaneous velocity, and $E_{0}(x)$ is the electric

field. In case of parallel plates geometry, the current is given by

$$
i=\frac{q \mu V_{\text {bias }}^{2}}{d^{2}}
$$

where $\mu$ is the mobility of the charge carrier, $V_{\text {bias }}$ is the voltage difference applied between the electrodes, and $d$ is the distance between the electrodes.

When the charge carrier lifetime $\tau_{e, h}$ becomes shorter than the transient time $t_{t r}=d / v$, the time that it takes for charge carrier to drift through detector, the transient form is affected by the

loss of charge due to trapping. When the trapped charge is not significantly distorting the electric field (i.e. before the crystal starts to polarize), the induced current for single charge carrier type with initial injected charge $q_{0}$ is given by

$$
i_{e, h}=\frac{q_{0} \mu V_{b i a s}^{2}}{d^{2}} e^{-\frac{t}{\tau_{e, h}}}
$$

Such change in charge carrier lifetime is expected when defects (e.g. radiation damage) are introduced.

The charge carrier velocity $v=d / t_{t r}$ can be also expressed by using an empirical formula by Caughey and Thomas [22] 


$$
v=v_{\text {sat }} \frac{\left(E / E_{c}\right)}{\left[1+\left(E / E_{c}\right)^{\beta}\right]^{(1 / \beta)}},
$$

where $v_{\text {sat }}$ is the saturation velocity, $E=V_{\text {bias }} / d$ is the electric field, $E_{c}$ is the critical field, and $\beta$ is an empirical fit parameter. Then the mobility $\mu$ is given by

$$
\mu=\frac{\mu_{0}}{\left[1+\left(E / E_{c}\right)^{\beta}\right]^{\left(1+\frac{1}{\beta}\right)}},
$$

where $\mu_{0}$ is the zero-field mobility that is calculated as

$$
\mu_{0}=\frac{v_{\text {sat }}}{E_{c}} \text {. }
$$

\section{Polarization}

Since no model describing the charge accumulation during polarization developed specifically for diamond was found, two models, multiple scales model describing the polarization in

$\mathrm{Cd}(\mathrm{Zn}) \mathrm{Te}$ detectors [23] and a simple model based on general equations for charge trapping [24], were used. In both models the electrode-diamond interface is assumed ohmic, high active trap concentration is assumed (i.e. $q N_{\text {trap }} \gg C_{\text {detector }} V_{\text {bias }}$ ) and increased trapping probability due to partial polarization is not taken into account. Only one charge carrier is considered at a time, while the other charge carrier is assumed to be immediately collected and not contributing to the polarization.

The crystal is fully polarized, when a sufficient charge is trapped to collapse the electric field in the detector. The minimum charge required to collapse the electric field is denoted by $Q^{*}$, whereas the time $t^{*}$ it takes to reach this critical state is obtained by numerically solving the time dependent accumulated charge $Q\left(t=t^{*}\right)=Q^{*}$.

\section{Multiple scales model}

The mathematical model for describing polarization in wide-bandgap semi-conductor has been developed by Bale and Szeles [23] mainly for $\mathrm{Cd}(\mathrm{Zn}) \mathrm{Te}$ applications. It was named multiple scales model for taking into account the differenttime scales for charge transport, trapping, and de-trapping.

The time dependence of charge accumulation in this model is given by

$$
Q(t)=\frac{q A_{\text {irr }} \phi E_{\alpha}}{v_{e f f} \epsilon_{E}} \begin{cases}1-e^{-\frac{v_{e f f} T}{\Lambda}}-\frac{v_{e f f} T}{\Lambda} e^{-\frac{L}{\Lambda},} & T \leq L / v_{e f f} \\ 1-\left(1+\frac{L}{\Lambda}\right) e^{-\frac{L}{\Lambda},} & T>L / v_{e f f}\end{cases}
$$

where $q$ is the elementary charge, $A_{i r r}$ is the area under irradiation, $\phi$ is the particle flux, $E_{\alpha}$ is the kinetic energy of one alpha-particle, $\epsilon_{E}$ is the energy required to produce an electron-hole pair, $L$ is the distance between parallel electrodes, $\Lambda$ describes the initial spatial distribution of accumulated charge, $T=t_{t r} t$ is time scaled with transient time in non-polarized detector, and $v_{\text {eff }}$ is the effective charge carrier speed reduced by the so called

'stop and go' process, where the carriers go trough multiple trapping in the process of traversing trough the detector:

$$
v_{e f f}=\frac{\tau_{e, h}}{\tau_{e, h}+\tau_{D}} \mu_{e, h} E,
$$

where $\tau_{e, h}$ is the electron or hole lifetime, $\mu_{e, h}$ is mobility, $E$ is the electric field, and $\tau_{D}$ is the mean detrapping time. 
The model was adjusted for diamond by choosing the mean free path of charge carrier as the characteristic length of spatial distribution of trapped charge

$$
\Lambda=v_{e, h} \tau_{e, h},
$$

where charge carrier velocity $v$ is given by Eq. (4). In addition to charge distribution, the minimum accumulated charge, $Q^{*}$, required to collapse the electric field is affected by the $\Lambda$.

In the original formulation $m b d a$ is the radiation length of X-rays and also the spatial distribution of generated charge carriers follows the exponential form $e^{-x / \Lambda}$. However, it does not hold in our case. The difference in spatial distribution of generated chargecarriers affects the location of the pinch point $x^{*}$, where the electric field has its minimum. This means that the equation for the minimum accumulated charge $Q^{*}$, that is required to collapse the electric field, might not be accurate. It is, however, used in the calculations.

$$
\text { redblack } Q^{*}=\frac{A \epsilon V_{\text {bias }}}{\Lambda}, \text { black }
$$

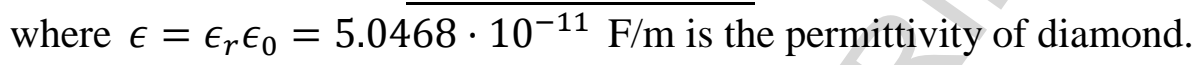

\section{Simple model}

An alternative model was derived from the classic formulation of time dependence on charge collection in presence of traps by Martini and McMath [24] originally for $\mathrm{Ge}(\mathrm{Li})$ and $\mathrm{Si}(\mathrm{Li})$ detectors. They presented an analytic solution for the case $t \leq t_{t r}$

$$
Q_{\text {coll }}=Q_{0} \frac{\tau_{\text {eff }}}{t_{t r}}\left[\frac{t}{\tau_{D}}+\frac{\tau_{e f f}}{\tau_{e, h}}\left(1-e^{-t / \tau_{e f f}}\right)\right],
$$

where $\tau_{e, h}$ is the lifetime of a charge carrier and $\tau_{e f f}$ is given by

$$
\tau_{e f f}=\frac{\tau_{e, h} \tau_{D}}{\tau_{e, h}+\tau_{D}}
$$

Then the charge in the detector at a given time is

$$
Q(t)=Q_{0}-Q_{\text {coll }} \text {, }
$$

where $Q_{0}$ is the injected charge. Assuming $\tau_{e, h}<\tau_{D}$ and $t_{t r}<\tau_{D}$ and requiring $Q_{\text {coll }}(t>$ $\left.\tau_{D}\right)=Q_{0}$, the charge remaining in detector is derived

$Q(t)$

$$
=Q_{0} \begin{cases}1-\kappa \frac{\tau_{e f f}}{t_{t r}}\left[\frac{t}{\tau_{D}}+\frac{\tau_{e f f}}{\tau_{e, h}}\left(1-e^{-t / \tau_{e f f}}\right)\right], & t \leq t_{t r} \\ 1-\kappa \frac{\tau_{e f f}}{t_{t r}}\left[\frac{t}{\tau_{D}}+\frac{\tau_{e f f}}{\tau_{e, h}}\left(1-e^{-t_{t r} / \tau_{e f f}}\right)\right], & t_{t r}<t \leq \tau_{D} \\ 0, & t>\tau_{D},\end{cases}
$$

where $\kappa$ is a dimensionless normalization constant

$$
\frac{1}{\kappa}=\frac{\tau_{e f f}}{t_{t r}}\left[1+\frac{\tau_{e f f}}{\tau_{e, h}}\left(1-e^{-t_{t r} / \tau_{e f f}}\right)\right] .
$$

The charge begins to accumulate, when the total injected charge also depends on time:

$$
Q_{0}(t)=\frac{q \phi A_{i r r} E_{\alpha}}{\epsilon_{E}} t .
$$

The total accumulated charge is obtained by convoluting the injected charge with the time

dependence of charge collection $f(t)=Q(t) / Q_{0}$ from Eq. (14)

$$
Q_{a c c}(t)=f(t) * Q_{0}(t)=\int_{0}^{\infty} f\left(t^{\prime}\right) Q_{0}\left(t-t^{\prime}\right) d t^{\prime},
$$

which is performed numerically.

The minimum trapped charge $\mathrm{Q} \stackrel{*}{\min }$ required to collapse the electric field is taken to be equal to the capacity of the detector, as a capacitor, to store charge: 
where $\epsilon$ is the permittivity of diamond, $A$ is the area of the parallel electrodes, and $d$ is the thickness of the diamond crystal. For instance, the minimum number of charge carriers required to collapse the electric fieldin a $300 \mu \mathrm{m}$ thick diamond used in this study is approximately $2 \cdot 10^{10}$ electrons/holes when the diamond is biased to $1 \mathrm{kV}$.

\section{Results and Discussion}

\section{State prior to polarization}

Figure 1. Measured and simulated TCT transients for $300 \mu \mathrm{m}$ thick non-irradiated diamond collecting electrons (a) and holes (b).

Figure 2. Measured and simulated TCT transients for $300 \mu \mathrm{m}$ thick diamond irradiated to $\mathbf{1 0}^{14}$ $\mathrm{p} / \mathrm{cm}^{2}$ collecting electrons (a) and holes (b).

Figure 3. Collected charge $Q_{\text {coll }}$ (area of current transient) for different irradiation fluences $\phi$. Scaled with the collected charge $Q_{\text {ref }}$ of non irradiated reference with thickness of $500 \mu \mathrm{m}$. Note that the y-axis has been broken for a better illustration of the data points.

The transients recorded within ten seconds of turning on the bias voltage and replicated with simulations are shown in Figs. 1 and 2. The measured signals have been post-processed to correct reflections andunder- or overshoots. The factor 2 difference in transient amplitudes in Fig. 1 and 2 is due to different gain in electronics. Electron and hole TCT measurements of non-irradiated detectors in Fig. ffig:nonirrad-simu show the expected rectangular pulse shape, indicating a constant electrical field. Simulated and experimental transients are in good agreement. In the beginning of the simulated transients a peak from the minority charge carrieris observed, which is filtered by the electronics in the measurements. In simulations it was dampened with an $50 \mathrm{Ohm}$ resistor in series with the detector. From Fig. 1 one can observe that the signal amplitudes for the negativevoltages get higher than the simulated ones, which could be explained by much more probable trapping of the electrons comparing to holes, or by increased mobility.

The charge collection efficiency (CCE) of the sensors can be obtained by numerically integrating over the current transient with respect to time and comparing the result to non-irradiated reference. Fig 3 shows the CCE measured in this manner for all of the irradiated sensors in a non-polarized state. The low fluence samples show no significant deterioration of CCE. The $300 \mu \mathrm{m}$ thick diamond shows a better charge collection than the reference, which is most likely caused bythe normal level of nitrogen impurities present in the $500 \mu \mathrm{m}$ thick non-irradiated reference diamond and is not related to irradiation. The high fluence samples show severely reduced CCE.

Figure 4. The measured charge transport velocities both (a) before and (b) after irradiation to $10^{14}$ $p / \mathrm{cm}^{2}$ are fitted with Equation (4) and compared to fits by Pomorski [20].

$$
\begin{aligned}
& \underset{(\mathrm{kV} / \mathrm{cm})}{E_{(\mathrm{cm}}} \mu_{2 / \mathrm{V}} \boldsymbol{v}_{\text {sat }}(\mathrm{cm} / \mathrm{s}) \beta_{\mathrm{R}}{ }^{2}
\end{aligned}
$$

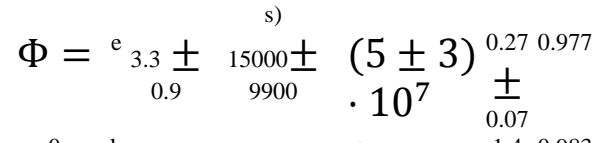

$$
\begin{aligned}
& \begin{array}{c}
0 \\
\mathrm{p} / \mathrm{cm}
\end{array} \quad \begin{array}{c}
\mathrm{h} \\
6.4 \pm 2000 \pm 80(1.28 \pm \\
0.2
\end{array}
\end{aligned}
$$




$$
\begin{aligned}
& \Phi=\begin{array}{c}
10^{7} \\
\pm 0.9 \\
\pm 0.9
\end{array} \quad \begin{array}{c}
(0.95 \\
\pm 0.05) \cdot \pm
\end{array} \\
& 10^{7} \quad 0.5 \\
& \begin{array}{c}
10^{14}{ }^{\mathrm{h}}{ }^{7.7} \pm 1520 \pm 90 \\
0.403) \cdot \pm
\end{array}
\end{aligned}
$$

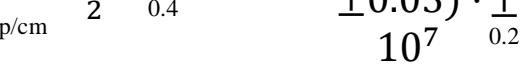

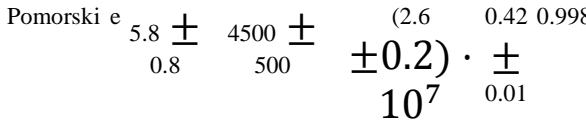

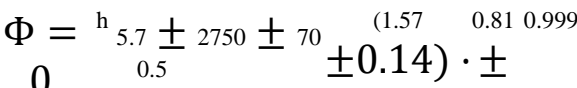

$$
\begin{aligned}
& \begin{array}{ccc}
0 & 0.5 & \pm 0.14) \cdot \pm \\
10^{7} & 0.01
\end{array}
\end{aligned}
$$

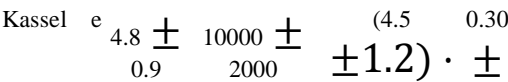

$$
\begin{aligned}
& 10^{7} \quad \frac{0.03}{1.54}
\end{aligned}
$$

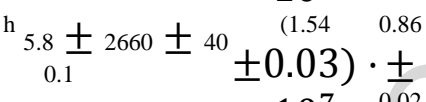

$$
\begin{aligned}
& 10^{7}
\end{aligned}
$$

Table 2. Charge transport properties for electrons (e) and holes (h) before and after irradiation compared to experimental values by Pomorski [20] and Kassel et al. [5]

The charge transport properties such as mobility and its high-field dependence were determined experimentally and used as parameters in the simulations. The drift velocity was obtained by dividing the transient time with the detector thickness. The transient time was measured from reaching $20 \%$ of the signal maximum on the rising edge to going below $15 \%$ of the signal maximum on the falling edge. In Fig. 4 the drift velocities of both non-irradiated diamond and diamond irradiated to fluence of $10^{14} \mathrm{p} / \mathrm{cm}^{2}$ are fitted with Eq. (4) and compared with the similar fits by Pomorski in his thesis [20]. In Table 2 the results of these fits are summarized. It is important to notice that in the case of the irradiated diamond, the detector is often partially polarized with the lower bias voltages $\left(V_{\text {bias }} / d<1 \mathrm{~V} / \mu \mathrm{m}\right)$. The zero field mobility $\mu_{0}$ and critical field $E_{c}$ are not very reliable. The saturation velocity,

however, is not very sensitive to low-field velocities and in the case of electrons it is significantly lowered from the value prior to irradiation.

In the simulations the electron transport properties of Pomorski fit was used, since it is in good agreement with the data in this study $\left(R^{2}=0.9678\right)$ and it takes into account higher electric fields than accessible in this study. The Pomorski fit is, however, not applicable to the holes transport and there the fit in this study is used. In the simulation of the irradiated diamond, the values for obtained for non-irradiated diamond were used for simplicity.

A clear state before polarization could be observed for the detectors with lower fluence of $10^{14} \mathrm{p} / \mathrm{cm}^{2}$. The electron and hole lifetimes $\tau_{e}=(23 \pm 2) \mathrm{ns}$ and $\tau_{h}=(80 \pm 30) \mathrm{ns}$, respectively, were found by fitting Eq. (3) to the reflection and undershoot corrected transient at bias voltage roughly corresponding to the electric field of $1 \mathrm{~V} / \mu \mathrm{m}$.

With the settings used in this study, a non polarized state could not be observed for the sensors irradiated to the higher fluence of $5 \cdot 10^{15} \mathrm{p} / \mathrm{cm}^{2}$. When radiation source was moved to a distance of $12 \mathrm{~mm}$ from the detector surface and theirradiated area was reduced to $50 \%$ of the

original with a grid-like collimator, a non polarized state could be briefly observed for

collecting holes with bias voltage $-1 \mathrm{kV}$ over $500 \mu \mathrm{m}$ thick detector. The duration of the current transient at $20 \%$ of the amplitude maximum was approximately $1 \mathrm{~ns}$. The charge carrier lifetime was estimated to be in order of $\tau_{h}=0.25 \ldots 0.5 \mathrm{~ns}$. With positive voltages (collecting electrons) a non polarized state could not be observed for detectors irradiatedto fluence of 5 . 


$$
10^{15} \mathrm{p} / \mathrm{cm}^{2} \text {. }
$$

\section{Polarization}

Figure 5. Current transients for electrons at different times after turning bias voltage on. The transient form follows the electric field in the $300 \mu \mathrm{m}$ thick diamond detector with TiW electrodes that has been irradiated to $10^{14}$ protons/cm ${ }^{2}$.

Figure 6. Evolution of electron transport properties with time within a sensor undergoing polarization. Charge collection efficiency (CCE) is the normalized integral of current transient, signal amplitude the maximum of the current transient.The observed hit rate is first limited by the set-up. The sudden drop indicates a drop in device efficiency. The detector used is same as in Fig. 5

A polarization effect, where charge collection efficiency drops suddenly, is observed for all diamond detectors after irradiation with protons and is absent in the same detectors before the irradiation. In Fig. 5 the time evolution of current transient is shown. Fig. 6 summarizes the evolution charge carrier collection properties during the polarization. The transient amplitude and charge carrier velocity decrease first slowly, while charge collection efficiency (CCE) and

observed rate remain constant. At a certain point CCE, transient amplitude, charge carrier velocity and observed rate drop abruptly. This happens when the critical amount of charge $\mathrm{Q}^{*}$ has accumulated in the detector volume.

Figure 7. Time $t^{*}$ to reach critical field $Q^{*}$ as a function of bias voltage for a) $300 \mu \mathrm{m}$ thick detectors irradiated to $10^{14}$ protons $/ \mathrm{cm}^{2}$, b) $500 \mu \mathrm{m}$ thick detector irradiated to $10^{14}$ protons $/ \mathrm{cm}^{2}$ and c) $500 \mu \mathrm{m}$ thick detectors irradiated to $5 \cdot 10^{15}$ protons $/ \mathrm{cm}^{2}$ compared to model calculations with simple model and multiple scales (MS) model discussed in section 2. The model parameters were either measured or retrieved from literature. The calculated values are scaled by factors of 20,8 and 2 for simple model, and $10-7$ and $4.5 \cdot 10^{-8}$ for multiple scales model in order to place them comfortably in the same figure with measurement results.

To investigate the polarization further, the time $t^{*}$ it takes for the irradiated detectors to polarize was measured under constant alpha-particle rate. The polarization times $t^{*}$ were obtained by fitting a straight line to the falling edge in the charge collection. The time, when the fitted line crosses noise threshold, was taken as $t^{*}$. The error bars in the measurements reflect the goodness of fit for the linear fit. This approach was chosen, because in some cases the polarization started developing already during voltage ramp-up and only the falling edge could be observed. For the high fluence samples only few measurement points were collected on the falling edge and this is reflected in the error bars. The polarization times grouped by detector

thickness and proton fluence are presented in Fig. 7 with example calculations of the computational models in section 2 . The experimental values found for fluence $10^{14} \mathrm{p} / \mathrm{cm} \quad 2$ in section 1 were used in the models together with literature values already discussed in section 4. De-trapping time $\tau_{D}=40 \mathrm{~s}$ was used. In the measurements, the polarization was observed to dissipate within 20 to 45 seconds after turning the bias voltage off. The used 40 seconds was shortest de-trapping time that consistently led to full polarization with both models. The model calculations for $500 \mu \mathrm{m}$ thick diamond irradiated to $5 \cdot 10^{15} \mathrm{p} / \mathrm{cm} \quad{ }^{2}$ were not successful.

The simple model is valid only for $\tau_{e, h}>\tau_{t r}$, which is no longer true.

The multiple scales model predicts considerably longer time for reaching polarization, $t^{*}=$ few years, than the measured few minutes. This is most likely due to pinch point for the electric

field extending outside the detector volume, which may result in erroneous results. The multiple scales model takes the spatial distribution of the trapped charge into consideration. In

the original formulation the spatial distribution depends primarily from the initial spatial distribution of the injected charge. This was adapted to diamond by taking the mean free path of the charge carrier as a measure of the charge spatial distribution. As the transient time of the 
charge carriers is close to the charge carrier lifetime, this might lead to unexpected behavior of the model.

The simple model predicts faster polarization than observed. This is most likely because the model does not take into account the spatial distribution of the trapped charge. Evenly distributed charge does not contribute to the collapse of the electric field in the same degree as locally clustered charge. The simple model result is closer to the measurement with $500 \mu \mathrm{m}$ thick detector.

The Fig 8 shows the ratio of polarization times for all samples and model calculations of the two models. The prediction of the multiple scales model differs significantly from all measurements. The simple model is similar to the measurement data in high field regime. At low field, the ratio of the polarization times is significantly different between different samples.

The samples seem to be divided into two groups: ones having nearly constant relation and others distinctive direct proportionality to electric field. The first group is similar to the simple model, where polarization is caused solely by neutral deep trap level in the bulk of the detector. The other group deviates significantly from the simple model at low field regime. This could indicate, for example, a non-neutral trap level in bulk or significant trapping in the diamond-metal interface in addition to the neutral defect level(s) in the bulk. The latter interpretation is supported by the fact that both $300 \mu \mathrm{m}$ samples fall into the category that deviates from the model prediction.

Figure 8. Relation between polarization time for holes and electrons as a function of bias voltage normalized with detector thickness for irradiated diamonds. Measurement data is compared to model calculations with the simple and multiple scales (MS) computational models.

The conditions assumed in Section 2 might not be valid. In particular the condition that the number of trapping defects in the detector volume is much larger than trapped charge required to collapse the electric field. The simulations are in agreement with the defect concentration of $10^{14} \mathrm{~cm}^{-3}$ for diamond irradiated to fluence of $10^{14}$ protons $/ \mathrm{cm}^{2}$. This means that the maximum trapped charge in the active volume of a $300 \mu \mathrm{m}$ thick detector is approximately

equivalent to $5 \cdot 10^{11}$ electrons. The minimum charge required to collapse the field is equivalent to $2 \cdot 10^{10}$ electrons. Clearly there is enough defects to collapse the field, but, to achieve the buildup of space charge necessary to collapse the electric field, nearly all traps might need to be filled.

\section{Conclusions}

Five high purity single crystal diamond-based detectors were investigated for space charge polarization using $\alpha$-TCT measurements and TCAD simulations. Strong enough polarization effect to collapse electric field under the high flux of injected charge from $\alpha$-particles in proton irradiated diamond was observed.

Estimated charge carrier lifetimes that favor the trapping of electrons in irradiated diamond are in accordance with previous studies $[5,6,7]$.

For the low fluence $\left(10^{14} \mathrm{p} / \mathrm{cm}^{2}\right)$ detectors, turning bias voltage periodically off for one minute and back on is an effective method for removing polarization. In this case full charge collection can be restored. In the measurement of minimum ionizing particles the injected charge per particle is much less than in this experiment. It follows, that the detector is expected to be operational for extended period of time before polarization occurs, especially, if high bias voltage is used.

For the high fluence $\left(5 \cdot 10^{14} \mathrm{p} / \mathrm{cm}^{2}\right)$ detectors, switching high voltage off and back on is not enough to restore charge collection efficiency and alternative methods for improving detector performance, such as filling traps or re-processing, are advised to be considered in high particle rate environment. 
There is need for development of numerical model to describe the polarization in diamond. The two models considered in this study are originally developed for other materials and do not take into account all the relevant aspects for diamond.

The models compared to the data are in better agreement with $500 \mu \mathrm{m}$ than $300 \mu \mathrm{m}$ thick detectors. This could indicate significant trapping at the diamond-metal interfaces.

\section{Acknowledgements}

Special thanks for the help provided by TOTEM collaboration, CMS-TOTEM Precision Proton Spectrometer community and the IRRAD-team. This project has received funding from the H2020 project AIDA-2020, GA no. 654168, Magnus Ehrnrooth Foundation, Svenska kulturfonden, Academy of Finland and Waldemar von Frenckell foundation.

\section{References}

[1] N. Venturi, A. Alexopoulos, M. Artuso, F. Bachmair, L. BÃăni, M. Bartosik, J. Beacham, H. Beck, V. Bellini, V. Belyaev, B. Bentele, P. Bergonzo, A. Bes, J.-M. Brom, M. Bruzzi, G. Chiodini, D. Chren, V. Cindro, G. Claus, J. Collot, J. Cumalat, A. Dabrowski, R. Dâ€TMAlessandro, D. Dauvergne, W. de Boer, C. Dorfer, M. Dunser, V. Eremin, G. Forcolin, J. Forneris, L. Gallin-Martel, M.-L. Gallin-Martel, K. Gan, M. Gastal, C. Giroletti, M. Goffe, J. Goldstein, A. Golubev, A. Gori $\AA_{j} e^{2}$, E. Grigoriev, J. Grosse-Knetter, A. Grummer, B. Gui, M. Guthoff, I. Haughton, B. Hiti, D. Hits, M. Hoeferkamp, T. Hofmann, J. Hosslet, J.-Y. Hostachy, F. HÃ¹/4gging, C. Hutton, J. Janssen, H. Kagan, K. Kanxheri, G. Kasieczka, R. Kass, F. Kassel, M. Kis, G. Kramberger, S. Kuleshov, A. Lacoste, S. Lagomarsino, A. L. Giudice, E. Lukosi, C. Maazouzi, I. Mandic, C. Mathieu, M. Menichelli, M. MikuÅ3/4, A. Morozzi, J. Moss, R. Mountain, S. Murphy, M. MuÅjkinja, A. Oh, P. Olivero, D. Passeri, H. Pernegger, R. Perrino, F. Pic, Re sults on radiation tolerance of diamond detectors, Nuclear Instruments and Methods in Physics Research Section A: Accelerators, Spectrometers, Detectors and Associated Equipmentdoi:https://doi.org/10.1016/j.nima.2018.08.038.

http://www.sciencedirect.com/science/article/pii/s01689 00218309914

[2] G. Antchev, P. Aspell, I. Atanassov, V. Avati, J. Baechler, V. Berardi, M. Berretti, E. Bossini, U. Bottigli, M. Bozzo, P. Broul⿵̃-m, A. Buzzo, F. Cafagna, M. Catanesi,

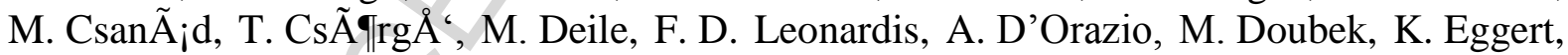
V. Eremin, F. Ferro, A. Fiergolski, F. Garcia, V. Georgiev, S. Giani, L. Grzanka, C. Guaragnella, J. Hammerbauer, J. Heino, A. Karev, J. KaÅ S. Lami, G. Latino, R. Lauhakangas, R. Linhart, M. LokajÃ-Ä• ek, L. Losurdo, M. L. Vetere, F. L. RodrÃ-guez, D. Lucsanyi, M. MacrÃ-, A. Mercadante, N. Minafra, S. Minutoli, T. Naaranoja, F. Nemes, H. Niewiadomski, T. Novak, E. Oliveri, F. Oljemark, M. Oriunno, K. Österberg, P. Palazzi, L. PaloÄ• ko, V. Passaro, Z. Peroutka, V. Petruzzelli, T. Politi, J. ProchÃ $\tilde{j}_{z k a}$ F. Prudenzano, M. Quinto, E. Radermacher, E. Radicioni, F. Ravotti, E. Robutti, C. Royon, G. Ruggiero, H. Saarikko, A. Scribano, J. Smajek, W. Snoeys, J. Sziklai, C. Taylor, N. Turini, V. Vacek, J. Welti, P., Diamond detectors for the TOTEM timing upgrade, Journal of Instrumentation 12 (03) (2017) P03007.

http://stacks.iop.org/1748-0221/12/i=03/a=P03007

[3] CMS-TOTEM Precision Proton Spectrometer, Technical report, CERN-LHCC-2014-021 TOTEM-TDR-003 CMS-TDR-13 (2014).

[4] Timing Measurements in the Vertical Roman Pots of the TOTEM Experiment, Technical report, CERN-LHCC-2014-020 TOTEM-TDR-002 (2014). 
[5] F. Kassel, M. Guthoff, A. Dabrowski, W. de Boer, Severe signal loss in diamond beam loss monitors in high particle rate environments by charge trapping in radiation-induced defects, physica status solidi (a) 213 (10) (2016) 2641-2649. doi:10.1002/pssa.201600185. http://dx.doi.org/10.1002/pssa.201600185

[6] M. Pomorski, E. Berdermann, W. de Boer, A. Furgeri, C. Sander, J. Morse, Ch arge transport properties of single crystal cvd-diamond particle detectors, Diamond and Related Materials 16 (4) (2007) 1066-1069, proceedings of Diamond 2006, the 17th European Conference on Diamond, Diamond-Like Materials, Carbon Nanotubes, Nitrides and Silicon Carbide. doi:https://doi.org/10.1016/j.diamond.2006.11.016.

http://www.sciencedirect.com/science/article/pii/s09259 63506004006

[7] V. Grilj, N. Skukan, M. JakÅ ¡ï̈‡, M. Pomorski, W. Kada, T. Kamiya, T. Ohshima, The evaluation of radiation damage parameter for cvd diamond, Nuclear Instruments and Methods in Physics Research Section B: Beam Interactions with Materials and Atoms 372 (2016) 161164. doi:https://doi.org/10.1016/j.nimb.2015.12.046.

http://www.sciencedirect.com/science/article/pii/s01685 $83 \times 16000021$

[8] W. Kada, N. Iwamoto, T. Satoh, S. Onoda, V. Grilj, N. Skukan, M. Koka, T. Ohshima, M. Jak $\AA_{j i} \mathrm{i} ̈+$, T. Kamiya, Co ntinuous observation of polarization effects in thin sc-cvd diamond detector designed for heavy ion microbeam measurement, Nuclear Instruments and Methods in Physics Research Section B: Beam Interactions with Materials and Atoms 331 (2014) 113-116, 11th European Conference on Accelerators in Applied Research and Technology. doi:https://doi.org/10.1016/j.nimb.2013.11.040.

http://www.sciencedirect.com/science/article/pii/s01685 $83 \times 14001347$

[9] Element Six Ltd., Kings Ride Park, Ascot, Berkshire SL5 8BP, UK. (2018).

https://www.e6.com

[10] PRISM, Micro fabrication laboratory at Princeton Institute for the Science and Technology of Materials (2018).

https://materials.princeton.edu

[11] Applied Diamond Inc., 3825 Lancaster Pike, Wilmington, DE 19805,USA (2018).

http://usapplieddiamond.com/

[12] Irradiation dosimetry results 2017 (2018).

https://ps-irrad.web.cern.ch/assets/doc/after/results/2

$017 /$ Sets-2017.html

[13] W. Shockley, Currents to conductors induced by a moving point charge, Journal of Applied Physics 9(10) (1938) 635-636. arXiv:https://doi.org/10.1063/1.1710367, doi:10.1063/1.1710367.

https://doi.org/10.1063/1.1710367

[14] S. Ramo, Currents induced by electron motion, Proceedings of the I.R.E. (1939) 584.

[15] Synopsys, Synopsys webpage, https://www.synopsys.com/, accessed: January, 2018 (2018).

[16] M. Brezeanu, Diamond schottky barrier diodes, Ph.D. thesis (2007).

https://www.repository.cam.ac.uk/handle/1810/226757

[17] A. Morozzi, Development and application of state-of-the-art device/circuit level tcad simulation tools for the optimization of innovative silicon-on-diamond (sod) semiconductor devices, Ph.D. thesis (2015/2016).

http://www.infn.it/thesis/thesis dettaglio.php?tid=12391

[18] T. Shimomura, Y. Kubo, J. Barjon, N. Tokuda, I. Akimoto, N. Naka, Quantitati ve relevance of substitutional impurities to carrier dynamics in diamond, Phys. Rev. Materials 2 
(2018) 094601. doi:10.1103/PhysRevMaterials.2.094601.

https://link.aps.org/doi/10.1103/PhysRevMaterials.2.094 601

[19] S. Dannefaer, A. Pu, D. Kerr, Po sitron annihilation study of vacancies in type iia diamonds illuminated with monochromatic light, Diamond and Related Materials 10 (12) (2001) 2113-2117. doi:https://doi.org/10.1016/S0925-9635(01)00489-7.

http://www.sciencedirect.com/science/article/pii/s09259 63501004897

[20] M. Pomorski, E lectronic properties of single crystal cvd diamond and its suitability for particle detection in hadron physics experiments, Ph.D. thesis (2008).

http://www-norhdia.gsi.de/publications/pomorski thesis final LQ.pdf

[21] $\bar{C}$. E. Nebel, Electronic properties of cvd diamond, Semiconductor Science and Technology 18 (3) (2003) S1.

http://stacks.iop.org/0268-1242/18/i=3/a=301

[22] D. M. Caughey, R. E. Thomas, Carrier mobilities in silicon empirically related to doping and field, Proceedings of the IEEE 55 (12) (1967) 2192-2193. doi:10.1109/PROC.1967.6123. [23] D. S. Bale, C. Szeles, Nature of polarization in wide-bandgap semiconductor detectors under high-flux irradiation: Application to semi-insulating ${ }^{2} d_{1-x} \operatorname{zn}_{x}$ Te, Phys. Rev. B 77 (2008) 035205. doi:10.1103/PhysRevB.77.035205.

https://link.aps.org/doi/10.1103/PhysRevB.77.035205

[24] M. Martini, T. McMath, Tra pping and detrapping effects in lithium-drifted germanium and silicon detectors, Nuclear Instruments and Methods 79 (2) (1970) 259-276. doi:https://doi.org/10.1016/0029-554X(70)90149-7.

http://www.sciencedirect.com/science/article/pii/002955 $4 \times 70901497$ 


\section{Highlights}

- Space charge polarization was studied with irradiated single crystal diamond sensors

- Full collapse of the electric field was observed for all irradiated samples

- To remove polarization in low fluence samples switching bias voltage on-off effective

- $\quad$ Polarization very likely cannot be explained by trapping in the bulk only 


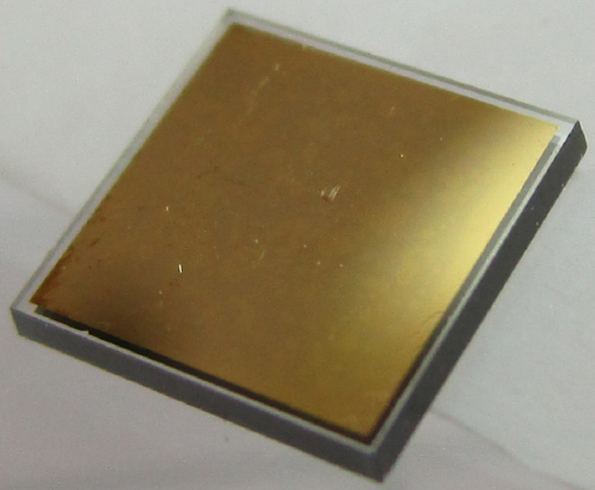

Figure 1 
(a)

\section{Current transients before irradiation}

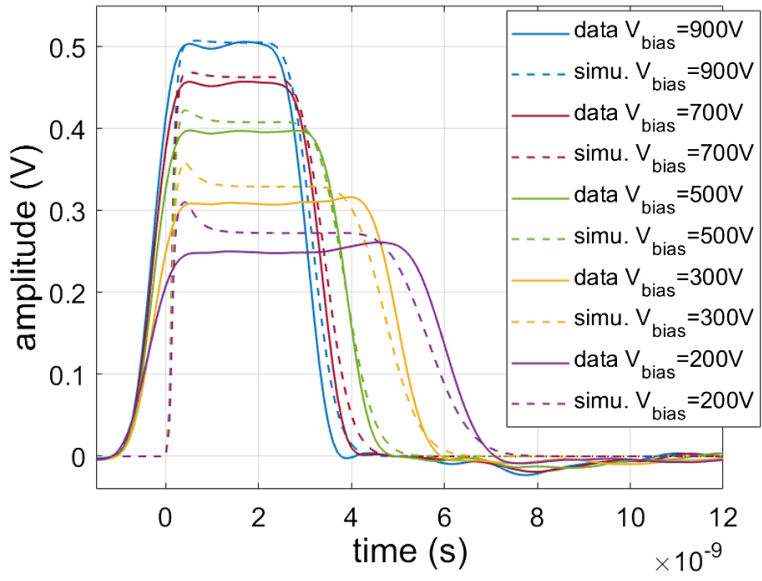

(b)

\section{Current transients before irradiation}

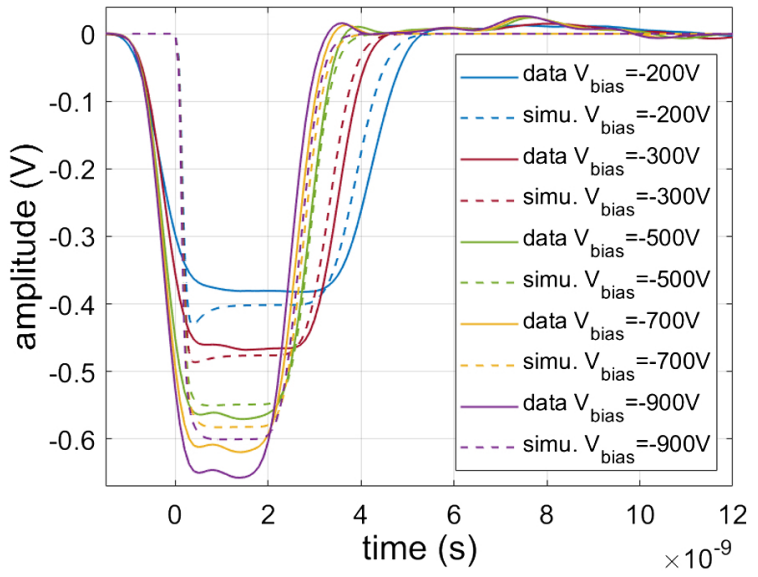


(a)

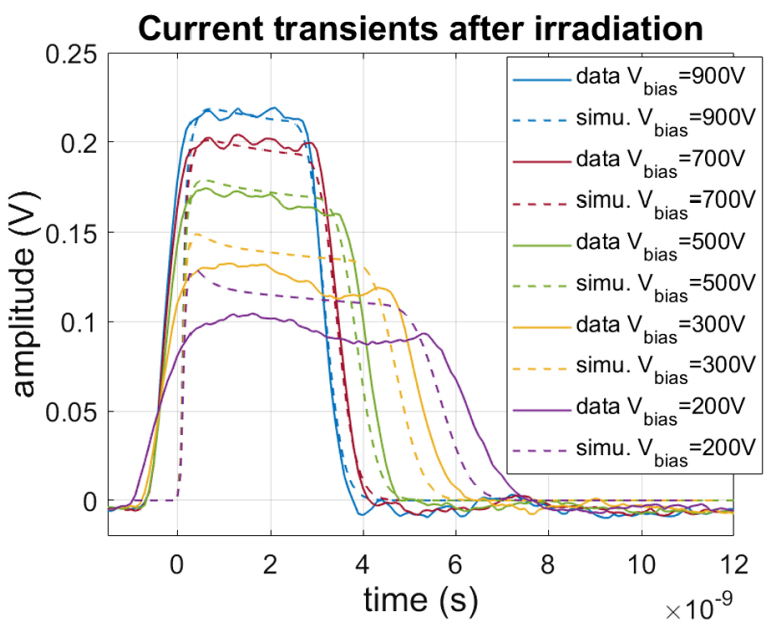

(b)

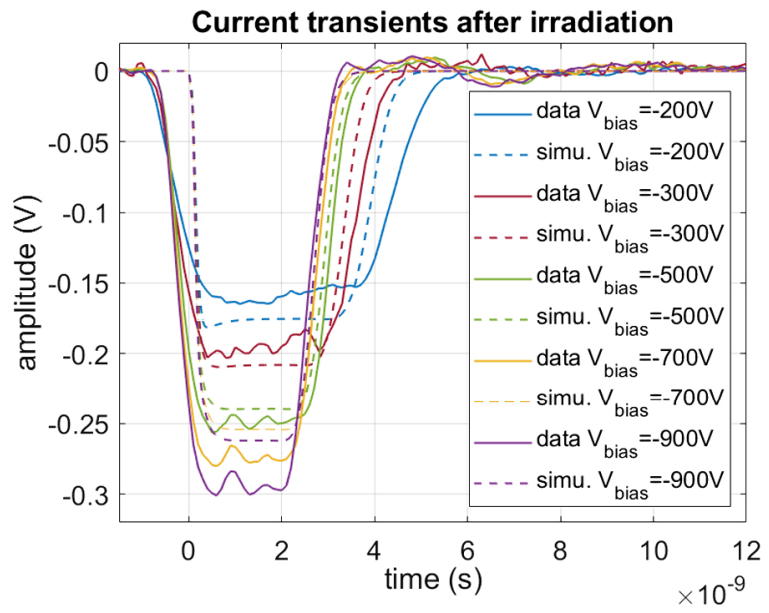




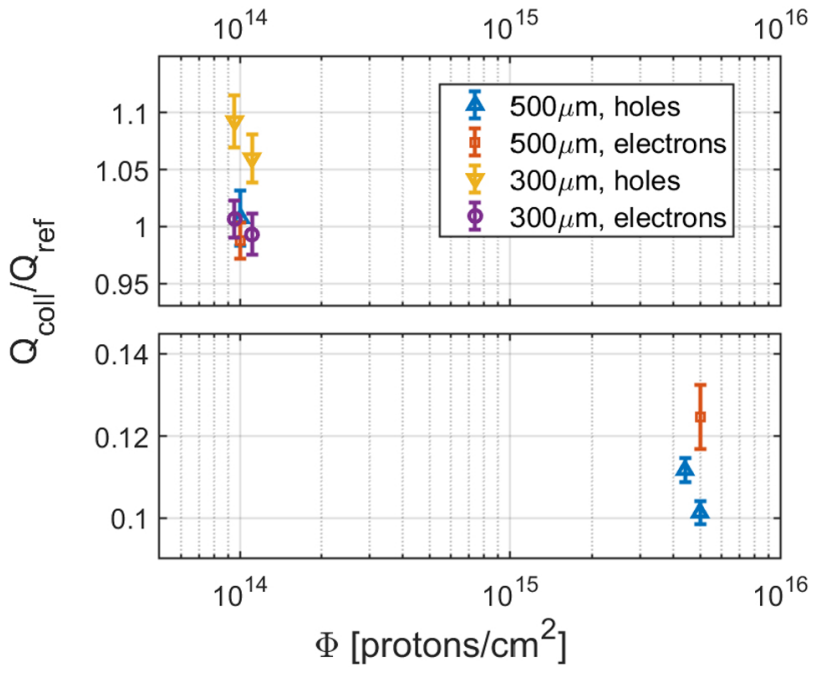

Figure 4 
(a)

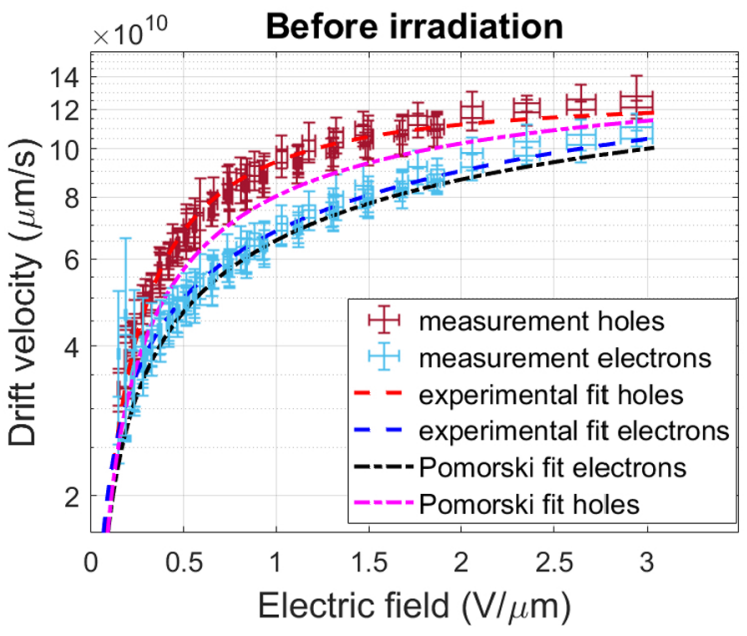

(b)

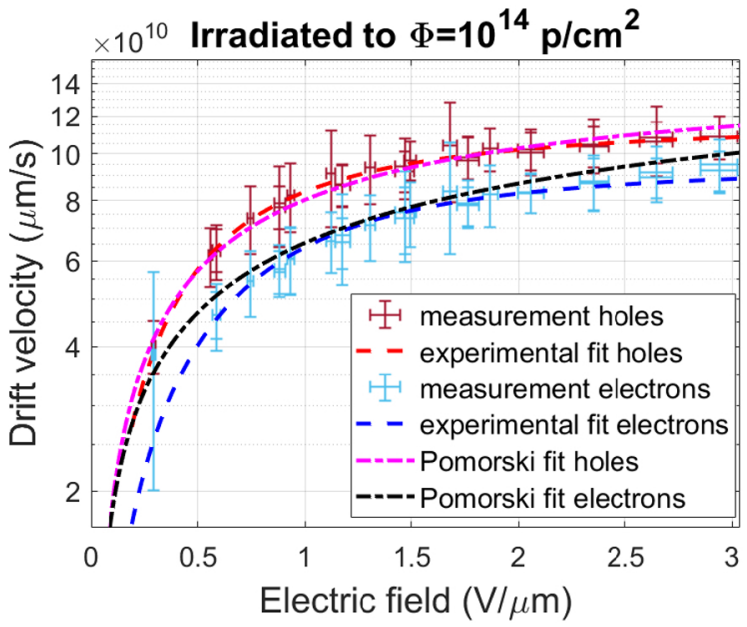


Current transients, $+500 \mathrm{~V}$

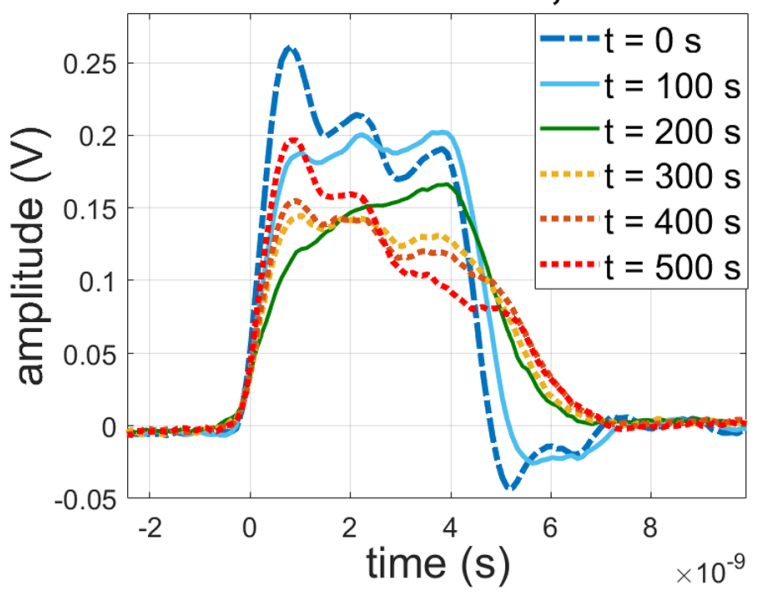

Current transients, $-500 \mathrm{~V}$

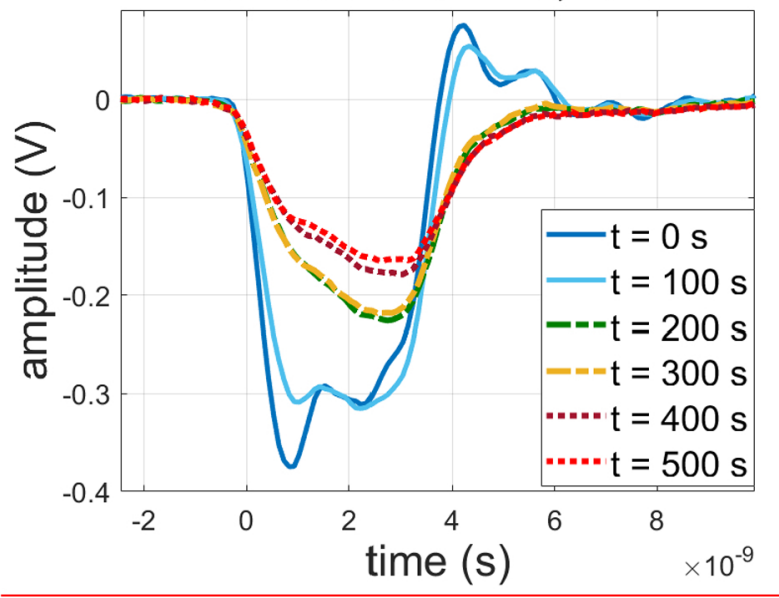



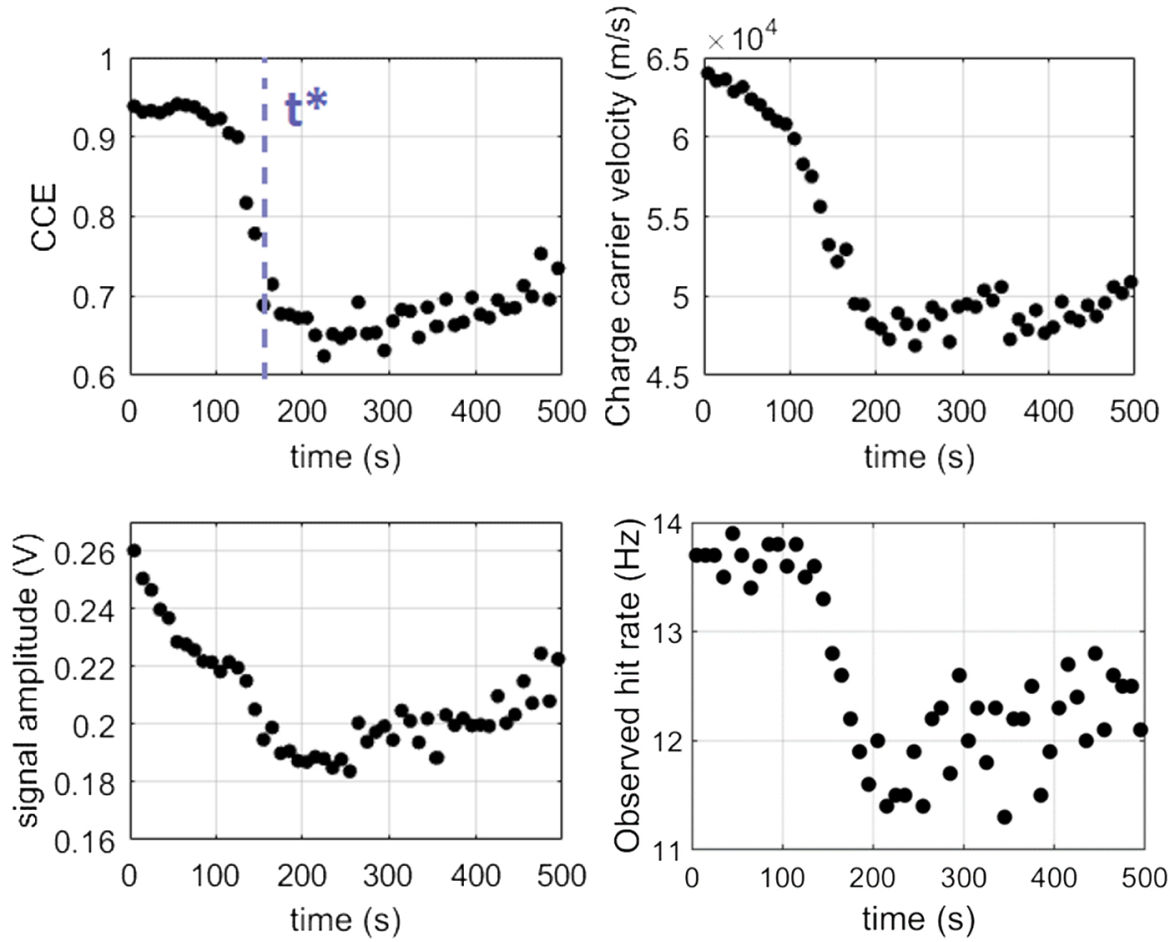

Figure 7 


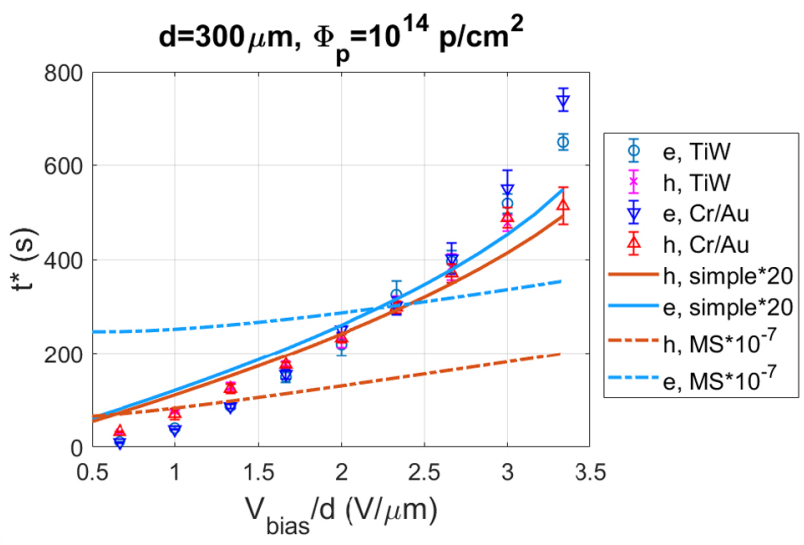

(a)

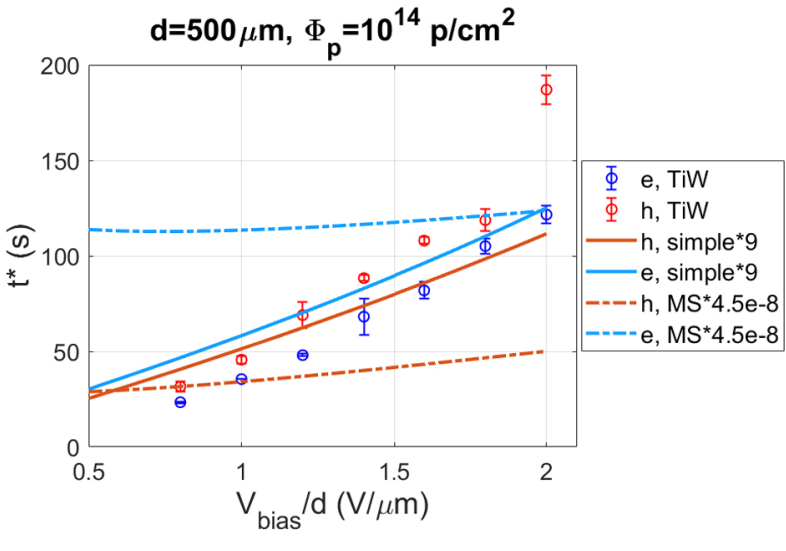

(b)

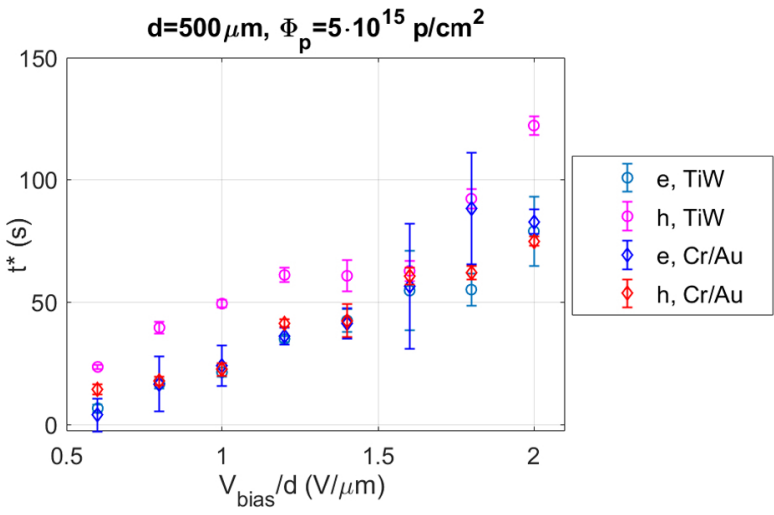

(c) 


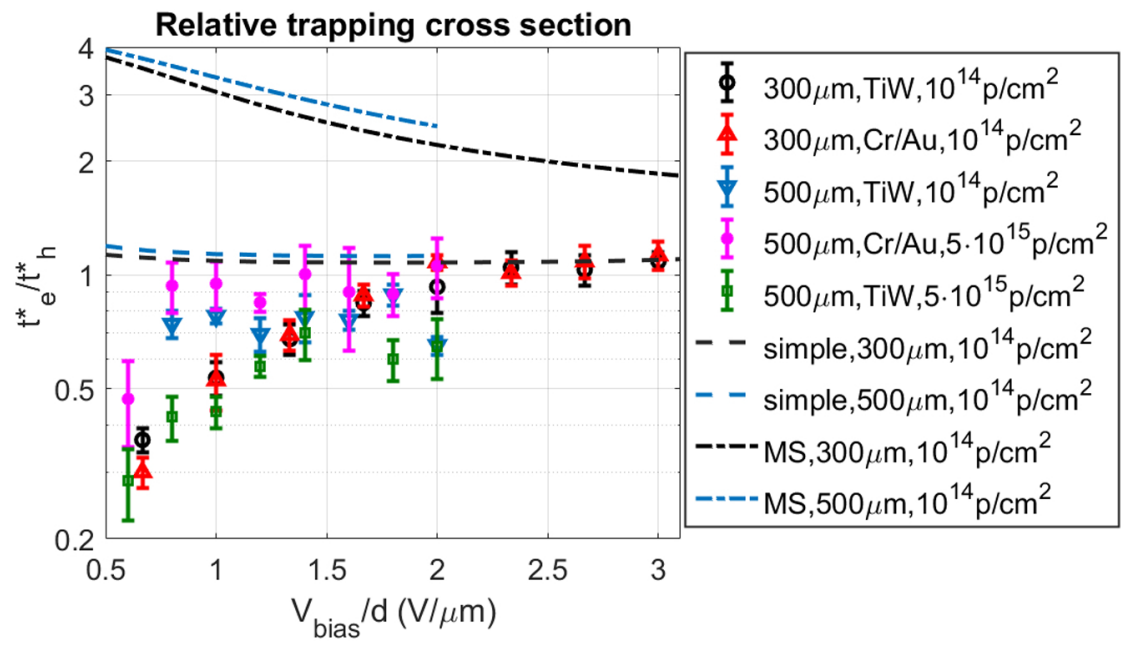

Figure 9 